\title{
La incidencia del entonamiento afectivo y la imitación en el involucramiento visual-social temprano
}

\author{
The Incidence of Affect fittunement and Imitation \\ in Early Social-Uisual Engagement \\ A incidência da sintonização afetiva e a imitação \\ no envolvimento visual-social prematuro
}

\begin{abstract}
Mariana Bordoni*, Silvia Español ${ }^{* *}$, Pablo De Grande ${ }^{* * *}$
${ }^{*}$ Consejo Nacional de Investigaciones Científicas y Técnicas (CONICET), Argentina, Instituto de Investigaciones Filosóficas (IIF) de la Sociedad Argentina de Análisis Filosóficos (SADAF), Buenos Aires, Argentina. ${ }^{* *}$ Consejo Nacional de Investigaciones Cientificas y Técnicas (CONICET), Argentina, Facultad Latinoamericana de Ciencias Sociales (FLACSO), Buenos Aires, Argentina. ${ }^{* * *}$ Universidad del Salvador, Buenos Aires, Argentina.
\end{abstract}

Doi: http://dx.doi.org/10.12804/ap134.3.2016.04

\section{Resumen}

Se realizó un estudio longitudinal cuasi-experimental cuyo objetivo es evaluar los efectos que tiene el entonamiento afectivo en el involucramiento visual infantil. Se analizó la pauta de mirada del bebé (frecuencia y duración promedio de mirada dirigida a la experimentadora), en el periodo de 6 a 12 meses, en tres condiciones de interacción social: entonamiento afectivo, imitación e interacción social prototípica. Los resultados de los modelos de regresión lineal muestran que la edad y la condición de interacción operan como factores con efectos independientes en los indicadores de mirada. Durante el periodo estudiado, tiene lugar un desarrollo general de la pauta de mirada más allá de la condición social de interacción: con la edad la frecuencia aumenta y la duración promedio de la mirada disminuye para todas las condiciones de interacción. El entonamiento afectivo generó menores valores de involucramiento visual del bebé. Se propone que el entonamiento afectivo es una pauta de reciprocidad que permite acompañar la actividad del bebé sin distraer su atención visual que puede, entonces, permanecer orientada al entorno.

Palabras clave: entonamiento afectivo; mirada; imitación; interacciones tempranas.

* Mariana Bordoni, Consejo Nacional de Investigaciones Científicas y Técnicas (CONICET), Argentina, Instituto de Investigaciones Filosóficas (IIF) de la Sociedad Argentina de Análisis Filosóficos (SADAF), Buenos Aires, Argentina; ${ }^{* *}$ Silvia Español, Consejo Nacional de Investigaciones Científicas y Técnicas (CONICET), Argentina, Área de Educación, Facultad Latinoamericana de Ciencias Sociales (FLACSO), Buenos Aires, Argentina; ${ }^{* * *}$ Pablo De Grande, Instituto de Investigación en Ciencias Sociales (IDICSO), Universidad del Salvador, Buenos Aires, Argentina.

La correspondencia concerniente a este artículo debe dirigirse a Mariana Bordoni, Sociedad Argentina de Análisis Filosóficos, Instituto de Investigaciones Filosóficas, Bulnes 642 (C1176ABL), Ciudad Autónoma de Buenos Aires, Argentina. Correo electrónico:mgbordoni@gmail.com

Cómo citar este artículo: Bordoni, M., Español, S., \& De Grande, P. (2016). La incidencia del entonamiento afectivo y la imitación en el involucramiento visual-social temprano. Avances en Psicología Latinoamericana, 34(3), 487-503. doi: http://dx.doi.org/10.12804/apl34.3.2016.04 


\section{fibstract}

A quasi-experimental longitudinal study was conducted. Its aim is to evaluate the effects of affect attunement in baby's visual engagement. The pattern of infant's looking (frequency and average duration gaze directed to the experimenter) was analyzed between 6 to 12 months in three social interaction conditions: affect attunement, imitation and prototypical social interaction. Linear regression models have shown that age and condition of interaction operate as factors with independent effects on infant gaze. With age, frequency increases and average duration of gaze decreases for all conditions of interaction. During the studied period, beyond the kind of social interaction, a general development of pattern of looking takes place. Regard to interaction condition, affect attunement generated the lowest values of infant's visual-engagement. Affect attunement is a pattern of reciprocity that generates intersubjective contact beyond looking. It allows to accompanying infant activity without distracting her visual attention which can then remain oriented to environment.

Keywords: affect attunement; gaze; imitation; early interaction.

\section{Resumo}

Realizou-se um estudo longitudinal quase-experimental sujo objetivo é avaliar os efeitos que tem a sintonização afetiva no envolvimento visual infantil. Analisou-se a pauta do olhar do bebé (frequência e duração média do olhar dirigido ao experimentador), no período 6-12 meses, em três condições de interação social: sintonização afetiva, imitação e interação social prototípica. Os resultados dos modelos de regressão linear mostram que a idade e a condição de interação operam como fatores com efeitos independentes nos indicadores do olhar. Durante o período estudado, tem lugar um desenvolvimento geral da pauta de olhar para além da condição social de interação: com a idade a frequência aumenta e a duração média do olhar diminui para todas as condições de interação. A sintonização afetiva gerou menores calores de envolvimento visual do bebé. Propõe-se que a sintonização afetiva é uma pauta de reciprocidade que permite acompanhar a atividade do bebé sem distrair a sua atenção visual que pode, então, permanecer orientada ao entorno.

Palavras-chave: sintonização afetiva; olhar; imitação; interações prematuras.

\section{El contacto psicológico temprano a través de la coincidencia}

Las interacciones tempranas entre el adulto y el bebé son fundamentales para el desarrollo sociocognitivo-emocional de los niños (Bornstein $\&$ Tamis-LeMonda, 2010) y se despliegan a través del uso de múltiples pautas de reciprocidad (Español, 2012; Fogel \& Garvey, 2007; Garvey \& Fogel, 2007; Ribas \& Seidl-de-Moura, 1999; Stern, 1985 [1991]; Villalobos, 2006). Entre las múltiples pautas conductuales de reciprocidad que ocurren en las interacciones tempranas, aquellas a través de las cuales se establece coincidencia, congruencia o semejanza entre las conductas de los participantes de la interacción han sido señaladas como comportamientos que permiten establecer un grado especial de mutualidad entre los individuos (Beebe, Rustin, Sorter, \& Knoblauch, 2003; Užgiris, 1999; Užgiris, Benson, Kruper, \& Vasek, 1989). Dentro de las actividades de establecimiento de coincidencias, en este estudio se seleccionaron el entonamiento afectivo y la imitación, y se analizaron los efectos que estas conductas tienen en la mirada del bebé.

\section{Entonamiento afectivo: coincidencias conductuales más allá de la imitación}

La imitación es la actividad de coincidencia por excelencia. En los últimos 40 años, el descubrimiento de la imitación neonatal (Maratos, 1973; Meltzoff \& Moore, 1977) y el reconocimiento de las imitaciones faciales y vocales recíprocas en 
las tempranas protoconversaciones adulto-bebé (Bateson, 1975; Trevarthen, 1998) han llevado a que distintos investigadores propongan que la imitación constituye una vía privilegiada y fundamental para el contacto social temprano porque establece una conexión directa y fácilmente accesible entre tú-yo (Kugiumutzakis, 1998; Maratos, 1998; Meltzoff, 2005 [2010]; Reddy, 2008). Sin embargo, en medio de la conmoción teórica que generaron los descubrimientos de la habilidad temprana de imitación, Stern (1985 [1991]) identificó otra actividad de coincidencia frecuente en las interacciones tempranas que hasta el momento no aparecía mencionada en las investigaciones de la época: el entonamiento afectivo. El entonamiento afectivo ocurre cuando, a través de un comportamiento diferente, la madre genera coincidencias con la pauta temporal (ritmo, duración y pulso), la intensidad (absoluta y su perfil) o la pauta espacial de la conducta de su bebé (Stern, 1985 [1991]; Stern, Hofer, Haft, \& Dore, 1985); por ejemplo, si el bebé sacude sus brazos o piernas rítmicamente, el adulto responde vocalizando en el mismo ritmo e intensidad. Este comportamiento materno fue observado por primera vez a partir de los 9 meses del bebé (Stern et al., 1985), pero trabajos empíricos posteriores encontraron episodios de entonamiento afectivo materno en momentos más tempranos del desarrollo (desde los 2 meses de edad) (Español, Bordoni, Carretero, Martínez \& Camarasa, 2013; Jonsson \& Clinton, 2006; Jonsson et al., 2001).

El entonamiento afectivo, en tanto es un comportamiento que implica la generación de semejanzas conductuales, es un fenómeno que se emparenta con la imitación y que puede ser fácilmente confundido con ella; sin embargo, éste constituye un modo de interacción social interpersonal específico que lleva a involucramientos sociales diferentes. Mientras que la imitación implica el establecimiento de una coincidencia global con la acción del modelo, el entonamiento afectivo implica la generación de una conducta diferente en la que se refunden las cualidades dinámicas del comportamiento infantil (intensidad, ritmo y espacio). Por otro lado, mientras que la imitación concentra el foco de atención de la díada en la acción realizada y compartida, el entonamiento afectivo transmite una comunión en la experiencia afectiva sentida (Stern, 1985 [1991]). Con el entonamiento afectivo se logra una experiencia interactiva que, más allá de las acciones concretas realizadas, conduce a la sintonía entre las formas dinámicas de la vitalidad (Sheets-Jonnstone, 2008; Stern, 2010), antes denominadas afectos de la vitalidad (Stern, 1985 [1991]). Las formas de la vitalidad es un concepto, recientemente acuñado por Stern (2010), que refiere al modo en que la mente humana representa la dinámica de la acción y la experiencia — propia y ajena - , la cual se expresa en el modo de actuar, y en los encuentros interpersonales brinda información crucial acerca del estado subjetivo del agente de la acción, distinta de la intención y de la meta de la acción (Di Cesare et al., 2014; Español, Martínez, Bordoni, Camarasa, \& Carretero, 2014; Stern, 2010).

\section{Entonamiento afectivo e imitación en la interacción temprana}

Desde su formulación, el concepto de entonamiento afectivo ha llevado a interesantes desarrollos teóricos en el ámbito clínico y académico (e.g. Beebe et al., 2003; Peixoto Junior \& Arán, 2011; Sheets-Johnstone, 2008; Stern, 1995; Wright, 2009); sin embargo, pese a la importancia que se le atribuye en el desarrollo infantil, los estudios empíricos realizados en el área son relativamente escasos. Todos ellos son estudios transversales-observacionales que se concentran en describir el comportamiento materno, registrar el momento de aparición en la interacción diádica y medir su frecuencia en interacciones espontáneas madre-bebé. Estos trabajos han documentado la ocurrencia frecuente del entonamiento afectivo en las interacciones tempranas madre-bebé (e.g. Jonsson \& Clinton, 2006), y en alguno de ellos se ha encontrado que, en relación con la edad, la frecuencia de 
entonamientos afectivos e imitaciones maternos muestran tendencias opuestas; la imitación es preponderante entre los 2 y 6 meses, y luego, a partir de esta edad, el entonamiento afectivo se vuelve más frecuente (Jonsson et al., 2001).

En comparación con el entonamiento afectivo, la imitación ha sido un área de estudio mucho más activa en psicología del desarrollo. En las últimas décadas, se ha profundizado, además, en los efectos que provoca el hecho de ser imitado. Se han realizado distintos estudios transversales de laboratorio que han logrado medir los efectos que tiene en el infante el ser imitado por un adulto. En tales estudios, se ha registrado que los bebés de entre 2 y 3 meses miran durante más tiempo la cara de su madre cuando ella los imita (Markova \& Legerstee, 2006); y se ha destacado que la imitación constituye una pauta de reciprocidad facilitadora para la interacción con bebés pequeños pues evita situaciones de sobreestimulación materna (Field, 1977). Por otro lado, estudios de preferencia con bebés mayores (de entre 9 y 14 meses) encontraron que la imitación de un adulto-experimentador se asocia a un involucramiento visual mayor en comparación al establecido con un experimentador que, sin imitarlo, genera conductas temporalmente contingentes a las del bebé (Agnetta \& Rochat, 2004; Meltzoff, 1990). El mismo efecto se ha observado en personas con autismo, quienes en otras condiciones de interacción suelen mirar muy poco a la cara del compañero de interacción (Dawson \& Galpert, 1990; Nadel, 2006).

Hasta el momento, no hay estudios sistemáticos que informen - como sí se ha hecho en el ámbito de la imitación - sobre la reacción que el entonamiento afectivo genera en el bebé, tampoco sobre su evolución en el tiempo y, mucho menos, sobre los efectos diferenciales que provocan en el bebé y en su involucramiento social con el adulto ambas actividades de coincidencia. La única referencia sobre el primer punto se encuentra en el trabajo original de Stern et al. (1985), en el cual informan que, frente a los entonamientos afectivos maternos, los bebés continúan con su comportamiento como si nada especial hubiera ocurrido. Frente a esto, los investigadores debieron recurrir a la perturbación de la interacción natural de la díada para confirmar que los bebés estuvieran sintiendo el entonamiento afectivo de la madre. Para hacerlo, seleccionaron algunas díadas que tuvieran entonamientos afectivos rutinarios y se les pidió a las madres que exageraran o que disminuyeran notablemente la intensidad de su conducta de entonamiento habitual para poder observar qué reacción provocaba en el bebé. En dichas situaciones se encontró que el bebé detenía rápidamente su acción para mirar a su madre, es decir que el bebé estaba esperando el entonamiento afectivo adecuado de su madre (Stern et al., 1985).

\section{La mirada en el involucramiento social temprano}

Los momentos de involucramiento social adulto-bebé se caracterizan por el uso de múltiples pautas de reciprocidad que permiten mantener activo el contacto social (sonrisas, vocalizaciones, contacto físico, expresiones faciales, etc.). Entre todas ellas, la pauta de mirada del bebé (su orientación y duración) ha sido uno de los comportamientos infantiles privilegiados en los estudios empíricos sobre cognición social temprana (Rochat \& Striano, 2002) y sobre los efectos de ser imitado (Agnetta \& Rochat, 2004; Markova \& Legerstee, 2006; Meltzoff, 1990). La mirada del bebé funciona como un regulador de la actividad de la madre en la interacción (Kaye \& Fogel, 1980; Stern, 1974). A lo largo del primer año de vida, los bebés cambian significativamente su patrón de atención visual mostrando un control más flexible y voluntario de su foco atencional (Richards, Reynolds, \& Courage, 2010). En contexto de interacción social, se ha observado que la mirada del bebé comienza a ser más móvil, rápida e intermitente, generando episodios de mirada al compañero menos duraderos pero más frecuentes y cambiando su foco de atención en 
distintas partes del rostro humano (D’Odorico \& Levorato, 1990; Kaye \& Fogel, 1980; Lewkowicz \& Hansen-Tift, 2012; Messer \& Vietze, 1984; Rochat, 2001 [2004]).

Como se ha señalado, los estudios revisados indican que la imitación provoca mayor atracción visual infantil, pero aún no se han hecho pruebas sistemáticas semejantes para el entonamiento afectivo. ¿El entonamiento afectivo y la imitación provocan efectos diferentes en la reacción visual del bebé? ¿Cambian estos efectos con la edad del bebé? Este trabajo se propone evaluar las diferencias que generan el entonamiento afectivo y la imitación del adulto en el involucramiento visual-social del bebé (pauta de mirada dirigida al compañero de interacción). En interacciones espontáneas, la imitación y el entonamiento afectivo son comportamientos adultos fugaces que ocurren esporádicamente; se ha observado que, durante el primer año de vida, tienen una frecuencia aproximada de un evento de imitación o entonamiento afectivo por minuto (Jonsson et al., 2001). Para poder evaluar las diferencias que provocan estas dos actividades de coincidencia en la reacción visual del bebé, se generaron situaciones artificiales de interacción social en las que se aumentó considerablemente la presencia de los comportamientos estudiados, siendo éstos la única pauta de reciprocidad utilizada para interactuar con el bebé, de manera tal que se pudiera hacer evidente la reacción que provoca cada actividad de coincidencia por separado en el bebé, durante la segunda mitad del primer año de vida. Así mismo, se incorporó una situación de interacción social espontánea, semejante a las interacciones sociales que adultos y bebés establecen naturalmente, para poder evaluar en qué medida cambia la respuesta del bebé cuando se encuentra en una situación de interacción social con entonamiento afectivo como única pauta de reciprocidad adulta con respecto a una situación en la que se le dan pautas de reciprocidad variadas, tal y como ocurre en una interacción social prototípica.

\section{Objetivos}

Este estudio se propone analizar los efectos que la interacción social con entonamiento afectivo tiene en el involucramiento visual-social del bebé (mirada del bebé dirigida al compañero de interacción) en el periodo 6-12 meses, en comparación con la reacción visual del bebé generada por la imitación (la actividad de coincidencia por excelencia) y con la reacción generada en una situación de interacción social prototípica.

Así mismo, se pretende identificar el efecto que provoca la edad del bebé en el involucramiento visual-social infantil a lo largo de la segunda mitad del primer año de vida, en los distintos modos de interacción social (interacción con entonamiento afectivo, interacción con imitación e interacción social prototípica).

Por último, se buscará distinguir en la evolución de la pauta de mirada la relación entre la frecuencia y la duración de la mirada del bebé dirigida al compañero en la interacción social con entonamiento afectivo, con imitación y en la interacción social prototípica.

\section{Método}

\section{Diseño y tipo de estudio}

Se realizó un estudio cuasiexperimental de diseño longitudinal (Gonzalez, Yu, \& Volling, 2012).

\section{Participantes}

La muestra quedó constituida por 16 bebés, de los cuales 7 son mujeres y 9 son varones. Se evaluó a los participantes en tres momentos en los que su edad promedio fue de 6 meses, 15 días (d.e. 7 días), 9 meses, 12 días (d.e. 8 días) y 12 meses, 18 días (d.e. 7 días), respectivamente.

La selección de los participantes se realizó a través de la técnica de bola de nieve, tomando como origen una lista de allegados al equipo de 
investigación en los que se identificaron familias con bebés menores de 6 meses y se los contactó telefónicamente. Se les informó brevemente sobre los objetivos generales y el procedimiento del presente estudio. Todas las familias participaron libre y voluntariamente, firmando un consentimiento informado. En términos de localización geográfica, se incluyeron familias residentes en el conglomerado urbano del Gran Buenos Aires ${ }^{1}$. Respecto al nivel educativo, los padres tenían estudios universitarios completos, con excepción de una familia en la que ambos padres tenían estudios secundarios completos.

En el curso de la investigación se produjeron bajas de participantes y exclusiones de material experimental: uno de los participantes abandonó el estudio por motivos de salud a los 9 meses; otro no fue incluido en el análisis debido a que fue diagnosticado con trastorno general del desarrollo (TGD) durante el tercer año de vida.

Ninguno de los demás bebés considerados en el análisis ha nacido prematuramente, ni ha estado en incubadora, ni ha sido diagnosticado con problemas evolutivos hasta la fecha.

\section{Procedimiento}

El estudio se propuso evaluar la evolución de la reacción visual-social de los bebés durante la segunda mitad del primer año de vida frente a dos actividades de coincidencia diferentes (entonamiento afectivo e imitación). Para ello, se realizaron sesiones controladas de interacción a los 6,9 y 12 meses de edad. Se estableció como criterio de selección la inclusión de bebés que hubieran cumplido dichas edades en los últimos 30 días, seleccionándose cantidades similares de varones y mujeres.

Para cada una de las edades seleccionadas, se realizaron sesiones bajo las condiciones de

1 El Gran Buenos Aires incluye la ciudad de Buenos Aires más los 14 partidos del conurbano bonaerense. interacción que eran de interés a esta investigación. Estas condiciones de interacción fueron interacción con entonamiento afectivo e interacción con imitación, y una sesión de interacción social prototípica. Esta última función como situación de control permitió comparar una interacción variada en recursos interactivos y en contingencia temporal (modo natural de establecer un contacto social) con las otras dos condiciones de interacción social (altamente estructuradas).

Las sesiones de interacción fueron realizadas en el hogar de cada niño. Durante los encuentros, la experimentadora interactuó con el bebé durante al menos 10 minutos en un espacio de la casa de $2 \mathrm{~m} \times 2 \mathrm{~m}$, aproximadamente, que fuera habitual para el bebé y en el cual hubiera silencio y buena iluminación para conseguir un buen registro audiovisual, el cual se realizó con una cámara filmadora fija. Se prefirió el piso como superficie de interacción, de modo que el movimiento de ambos no se viese impedido por la ubicación del niño en alguna postura impuesta (como en una sillita) y para favorecer el encuentro cuerpo-a-cuerpo, ya que hay acciones y experiencias afectivo-emocionales que requieren del uso del cuerpo completo y no sólo de la expresión facial. En cada momento evolutivo, las tres sesiones de interacción fueron espaciadas semanalmente y todas ellas fueron acordadas con los padres del bebé según sus horarios de sueño y alimentación. También se atendió al buen estado de salud y de predisposición del bebé; siempre que el bebé manifestó malestar o incomodidad persistentes se suspendió la filmación y se reprogramó la sesión de interacción para los días posteriores.

\section{Instrumento}

Durante las sesiones el estímulo utilizado para evaluar el comportamiento de los bebés fue la interacción con la experimentadora. Las sesiones y el comportamiento de la experimentadora se estructuraron del siguiente modo. 
La condición de interacción con entonamiento afectivo comenzó con 3 minutos de juego libre para establecer un "buen clima" de interacción. Luego continuó con 5 minutos durante los cuales la experimentadora ejecutó conductas en las que hizo coincidir la cantidad, la intensidad y la pauta temporal de la conducta del bebé, pero en una modalidad conductual diferente. Por ejemplo, cuando el bebé vocalizó, la experimentadora le hizo toques en el cuerpo; cuando el bebé realizó movimientos corporales, la experimentadora respondió con vocalizaciones. Por último, se establecieron 2 minutos de cierre con juego libre. En la tabla 1 se resumen las respuestas de la experimentadora a las conductas del bebé en las condiciones de interacción con imitación y de interacción con entonamiento afectivo.

La condición de interacción con imitación tuvo una estructura similar a la de entonamiento afectivo. Se inició con 3 minutos de juego libre, se continuó con 5 minutos en los cuales la experimentadora replicó globalmente las acciones y comportamientos detectados del bebé en la misma modalidad conductual, incluyendo los movimientos corporales, las vocalizaciones, el uso de objetos y los desplazamientos. Finalmente, se establecieron 2 minutos de cierre con juego libre.

La condición de interacción social prototípica se utilizó como primer encuentro para lograr que el bebé se sintiera cómodo y en confianza con la experimentadora y para que ella se familiarizara con el repertorio conductual del bebé. Consistió en encuentros de 10 minutos de duración en los que la experimentadora, buscando mantener un contacto social agradable, se mantuvo receptiva y participativa a las actividades "propuestas" por el niño; sin embargo, también generó iniciativas propias según un conjunto de pautas de invitación a la interacción, que incluyó formas de juego frecuentes en las interacciones naturales adulto-bebé. Estos fueron, por ejemplo, juegos de expectativa de acercarse y alejarse al niño; esconderse y salir de detrás de un objeto; cantar canciones infantiles; realizar chasquidos con la boca o los dedos; el golpeteo rítmico de objetos.

Todos los bebés participaron en todas las condiciones de interacción. El orden de las sesiones de interacción con imitación y de interacción con

Tabla 1

Respuestas pautadas a las conductas del bebé en las condiciones de imitación y entonamiento afectivo

\begin{tabular}{lll}
\hline \multirow{2}{*}{ Conducta del bebé } & \multicolumn{1}{c}{ Imitación } & \multicolumn{1}{c}{ Respuesta } \\
\cline { 2 - 3 } Gatea. & Gatea. & $\begin{array}{l}\text { Entonamiento afectivo } \\
\text { gateo. }\end{array}$ \\
\hline $\begin{array}{l}\text { Patea, aplaude, sacude alguna parte la velocidad y el ritmo del } \\
\text { de su cuerpo. }\end{array}$ & $\begin{array}{l}\text { Patea, aplaude o sacude la misma parte del } \\
\text { cuerpo. }\end{array}$ & $\begin{array}{l}\text { Vocaliza a la velocidad y el ritmo de los } \\
\text { movimientos del niño. }\end{array}$ \\
\hline $\begin{array}{l}\text { Patea, sacude, golpea, rasca o tira } \\
\text { un objeto. }\end{array}$ & $\begin{array}{l}\text { Patea, sacude, golpea, rasca o tira un ob- } \\
\text { jeto igual. }\end{array}$ & $\begin{array}{l}\text { Vocaliza a la velocidad y el ritmo de los } \\
\text { movimientos del niño. }\end{array}$ \\
\hline $\begin{array}{l}\text { Detiene su movimiento tomando una } \\
\text { postura peculiar ("como estatua"). }\end{array}$ & $\begin{array}{l}\text { Adopta misma postura corporal todo el } \\
\text { tiempo que el bebé quede detenido en } \\
\text { dicha posición. }\end{array}$ & $\begin{array}{l}\text { Hace una vocalización suave y sostenida } \\
\text { que dure todo el tiempo que el bebé esté } \\
\text { quieto en dicha posición. }\end{array}$ \\
\hline Vocaliza. & $\begin{array}{l}\text { Vocaliza. } \\
\text { Tose/estornuda/bosteza. }\end{array}$ & $\begin{array}{l}\text { Toca el cuerpo del bebé con la duración, el } \\
\text { ritmo y la intensidad sentida de las voca- } \\
\text { lizaciones. }\end{array}$ \\
\hline
\end{tabular}


entonamiento afectivo fue contrabalanceado (la mitad de los bebés primero fue filmada en imitación y la otra mitad en entonamiento afectivo).

Como pauta general, en todos los casos se procuró sostener una interacción confortable con el bebé, intentando mantener la continuidad de la interacción como si se tratara de una interacción no artificial.

\section{Materiales}

Para cada sesión de interacción se contó con un conjunto de objetos puestos a disposición del bebé para su exploración, que fueron acordes con las capacidades motrices y los intereses lúdicos típicos de cada edad. En los primeros meses de vida, el bebé explora su cuerpo y los objetos de su entorno a través del juego sensorio-motor; entre los 9 y los 12 meses surge el juego funcional, en el que el niño explora el uso convencional de los objetos de su entorno, como cucharas, peines, regaderas (Español, 2004). Por tal motivo se incluyeron objetos atractivos para la exploración sensorio-motora (que se puedan golpear, chupar, morder) y objetos con usos convencionales conocidos para los niños de nuestra cultura (cucharas, vasos). A los 6 meses se utilizaron dos recipientes plásticos con tapa transparente de base rectangular y dos posavasos circulares de mimbre. A los 9 meses se agregaron dos cuadrados de goma eva y dos vasos de plástico. A los 12 meses se incorporaron cuatro cucharitas de acrílico transparente coloreado.

\section{Codificación del material observacional}

Se codificó la mirada del bebé dirigida a la experimentadora cada vez que el niño miró de forma directa y focalizada a la experimentadora. Se registró el momento de inicio y de finalización de cada mirada. Se excluyó de codificar todos los casos en los que no se pudo distinguir con claridad si el bebé estaba mirando a la experimentadora o al objeto que ella estuviera manipulando.
Se realizó la codificación sobre los minutos correspondientes a las condiciones experimentales de entonamiento afectivo y de imitación, y se seleccionaron y codificaron 5 minutos de las sesiones de interacción social prototípica ubicados a partir del minuto 4 para obtener tramos de igual duración en todas las condiciones. La codificación de los videos fue realizada en el programa Anvil 5.1.9. (Kipp, 2008). Se midió la confiabilidad interjueces con el mismo programa para el $20 \%$ del material audiovisual. Se obtuvieron coeficientes kappa de entre 0,81 y 0,85 .

\section{Análisis estadísticos del material observacional}

A partir del material obtenido en las sesiones experimentales, se observó la modificación del involucramiento visual-social de los bebés a lo largo del tiempo (medido en frecuencia y duración promedio de mirada dirigida a la experimentadora) en las tres condiciones de interacción (interacción con entonamiento afectivo, interacción con imitación e interacción social prototípica).

La frecuencia de mirada del bebé dirigida a la experimentadora fue definida como la cantidad de veces que el bebé se orientó visualmente hacia la experimentadora de manera directa y focalizada durante la sesión de interacción. La duración promedio de mirada del bebé dirigida a la experimentadora se definió como la suma total de segundos que el bebé sostuvo la mirada a la experimentadora sobre la cantidad de veces que la miró durante la sesión de interacción.

Sobre estos indicadores se realizó, en primer lugar, un análisis descriptivo de sus valores de tendencia central. Se presentan, además, gráficos de dispersión con su coeficiente $r$ por condición de interacción y edad, y los valores de promedio y desvío estándar (d.e.) para cada edad y condición.

En segundo lugar, se realizó un análisis inferencial con modelos de regresión lineal para determinar si los valores de los factores intervinientes en 
el análisis (edad y condición de interacción) son compatibles con la hipótesis de que cada uno de ellos - en forma independiente - afecta significativamente a los niveles de mirada, así como también para establecer la fuerza de los efectos causados por cada uno de ellos.

Los modelos fueron calculados utilizando la funcionalidad de modelos mixtos de SPSS. Esta herramienta permite elaborar modelos de regresión lineal con datos donde la varianza no se distribuya en forma normal, lo que resultó pertinente a nuestros datos debido a la correlación esperable entre las sucesivas observaciones de cada sujeto. De este modo, en los estimadores de confianza se tuvo en cuenta la correlación en los errores de los modelos de regresión por tratarse de muestras repetidas por ser un tipo de estudio longitudinal.

\section{Resultados}

En primer lugar, se presenta un análisis descriptivo correspondiente a los indicadores de involucramiento visual-social (frecuencia y duración promedio de mirada del bebé dirigida a la experimentadora), según edad y condición correspondientes a las 144 sesiones de interacción registradas. Luego se presentan dos modelos de regresión lineal con la finalidad de comparar los efectos estimados de manera independiente de la edad y de la condición de interacción sobre éstos.

\section{Frecuencia de mirada del bebé dirigida a la experimentadora}

Como tendencia general, se destaca una mayor frecuencia de mirada en las condiciones de interacción social con imitación $(25,23$ miradas, d.e. $=8,89)$ y de interacción social prototípica $(24,98$ miradas, d.e. $=9,35)$ en comparación con las sesiones de interacción con entonamiento afectivo $(18,94$ miradas, d.e. $=9,58)$.

En la figura 1 se observa la relación entre la frecuencia de mirada dirigida a la experimentadora y la edad de los bebés en cada una de las condiciones de interacción (cada punto representa la frecuencia de mirada de cada bebé participante en la sesión de interacción específica). En el caso de las sesiones de interacción con imitación, se constata que la frecuencia aumenta con la edad de los bebés, con un coeficiente de correlación $r=0,588(p<0,01)$. El promedio de veces en las que el bebé miró a la experimentadora en las sesiones de interacción con imitación a los 6 meses fue de 19,1 veces, llegando a las 31,8 veces en las correspondientes a los 12 meses. Por su parte, en la condición de interacción con entonamiento afectivo la correlación con la edad es también positiva pero de menor fuerza, con un coeficiente de correlación $r=0,346(p<0,05)$. Finalmente, la condición de control (interacción social prototípica) también muestra un aumento en la cantidad de veces que el bebé fijó su mirada en la experimentadora pero con un nivel de asociación intermedio respecto a las dos condiciones recientemente presentadas $(r=0,449 ; p<0,01)$.

\section{Duración promedio de mirada dirigida a la experimentadora}

La duración promedio de mirada del bebé dirigida a la experimentadora por sesión de interacción fue mayor en las condiciones de interacción social con imitación $(3,91$ segundos, d.e. $=1,40)$ y de interacción social prototípica (4,53 segundos, d.e. $=1,93)$ en comparación con las sesiones de entonamiento afectivo (3,59 segundos, d.e. $=1,93)$.

En la figura 2 se observa la relación entre la duración promedio de mirada y la edad de los bebés en cada una de las condiciones de interacción analizadas.

A diferencia de lo registrado para la frecuencia de mirada, a medida que aumenta la edad se observa una reducción en la duración promedio de la mirada, es decir que los bebés, a medida que fueron creciendo, en promedio, fijaron la mirada en la experimentadora por menos tiempo en cada oportunidad. 
a. Imitación

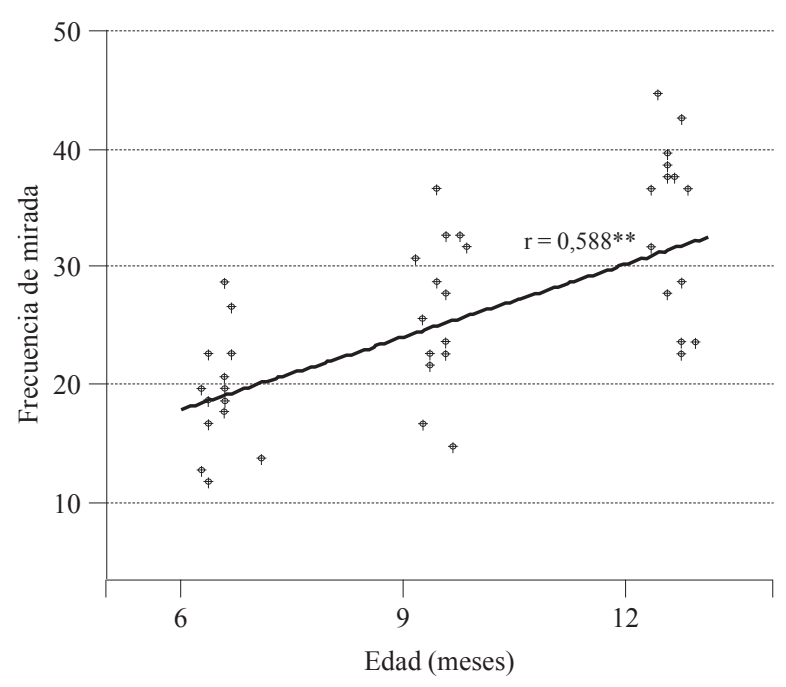

b. Entonamiento afectivo

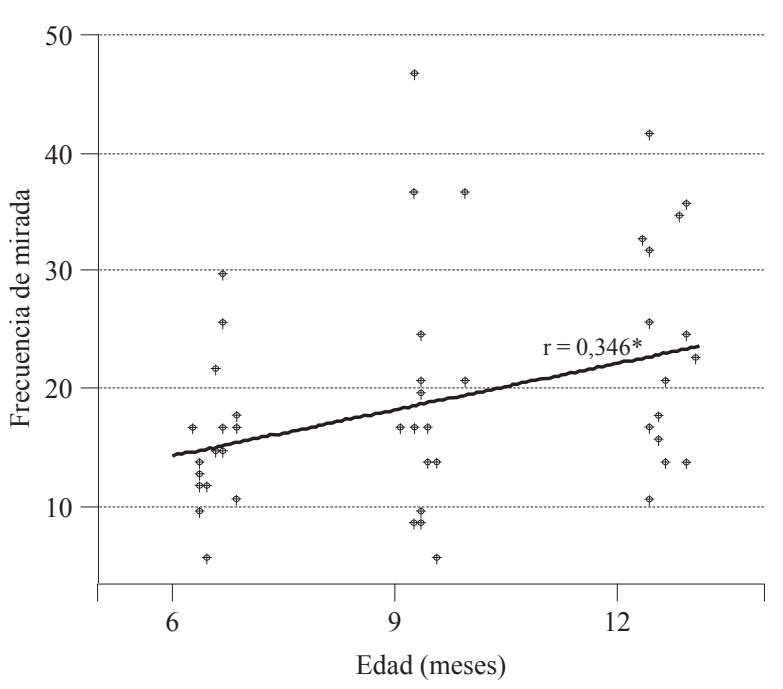

c. Interacción social prototípica

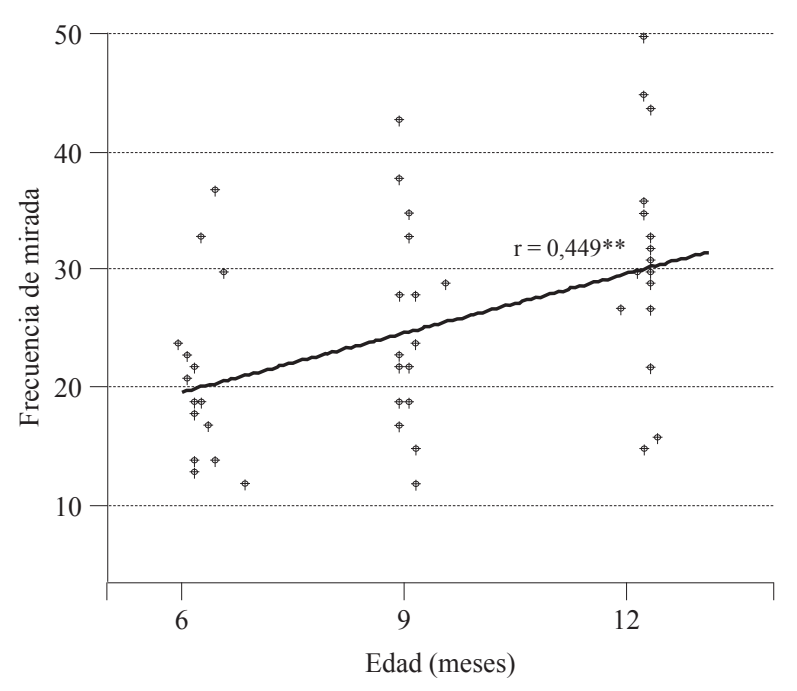

Figura 1. Frecuencia de mirada dirigida a la experimentadora por sesión de interacción para las condiciones de interacción con imitación (a), con entonamiento afectivo (b) y de interacción social prototípica (c), según edad del bebé (en meses). Cada punto en el gráfico representa la frecuencia de mirada de cada bebé participante. ${ }^{*} p<0,05$; $*^{* *} p<0,01$

El coeficiente de correlación para esta asociación en el caso de la imitación fue de $r=-0,547$ $(p<0,01)$. En valores absolutos, el promedio de duración de mirada para la condición de interacción con imitación fue de 5 segundos a los 6 meses, descendiendo hasta los 3,1 segundos a los 12 meses.
En la condición de entonamiento afectivo (figura $2 b$ ) es posible ver una tendencia similar, con duraciones promedio que van desde los 4,4 segundos a los 6 meses hasta los 3,05 segundos a los 12 meses. La asociación entre edad y duración promedio de mirada también es significativa en 
términos de coeficiente de correlación, pero es de menor intensidad que en la condición de interacción con imitación $(r=-0,289 ; p<0,05)$.

Por último, en la interacción social prototípica se advierte una tendencia de igual sentido que para las dos condiciones previas, con una intensidad en la relación intermedia, donde $r=-0,416(p<0,01)$ (figura 2c). Las duraciones de mirada para esta condición fueron levemente más largas que para las demás condiciones de interacción, siendo en promedio de 5,2 segundos a los 6 meses y de 3,3 segundos a los 12 meses de edad. a. Imitación

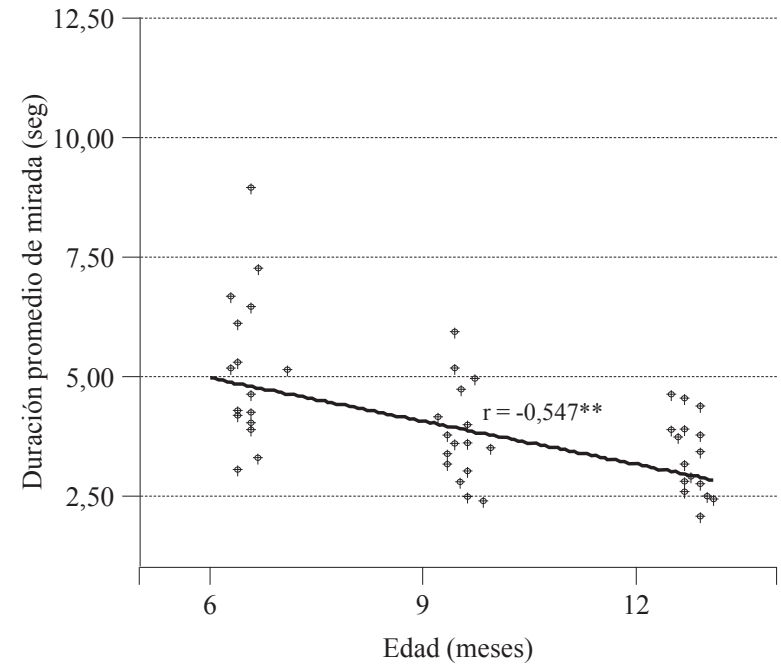

b. Entonamiento afectivo

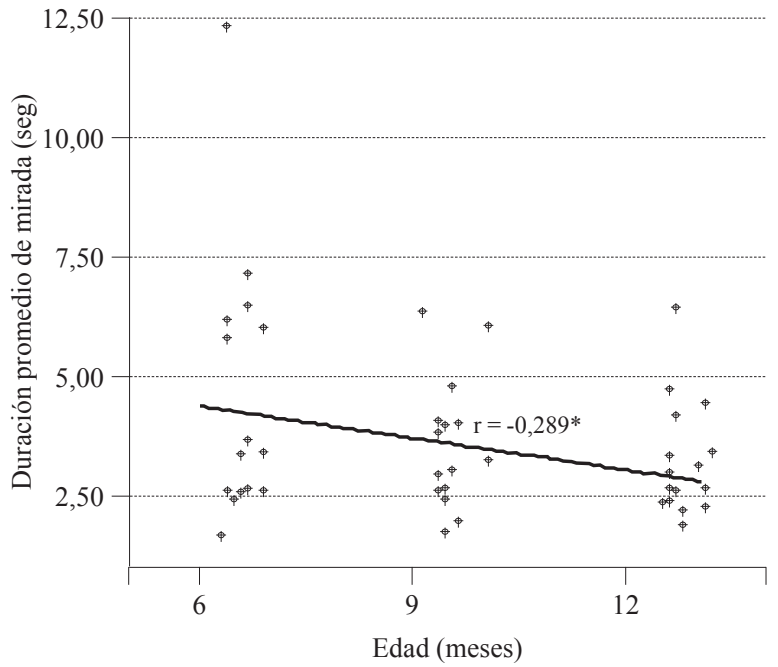

c. Interacción social prototípica

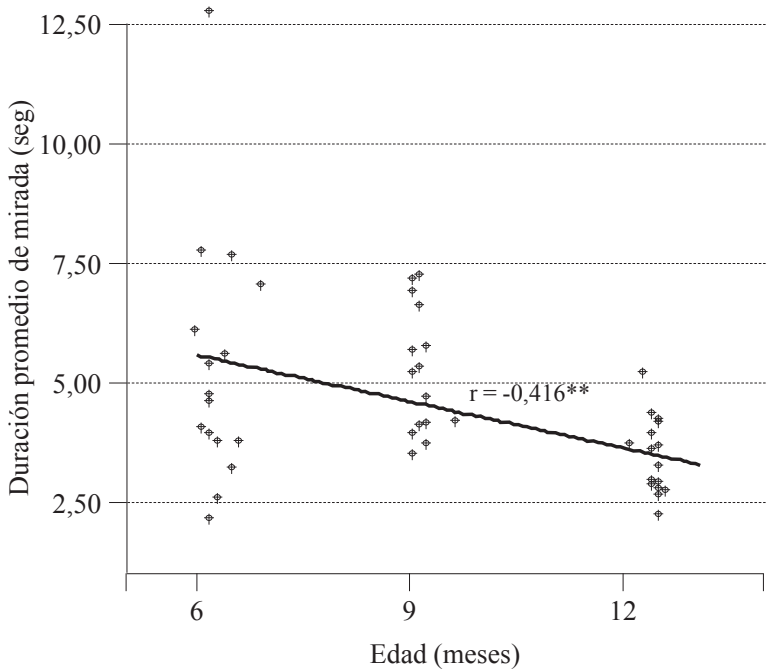

Figura 2. Duración promedio de mirada dirigida a la experimentadora para las condiciones de interacción con imitación (a), con entonamiento afectivo (b) y de interacción social prototípica (c), según edad del bebé (meses). Cada punto en el gráfico representa la duración promedio de mirada durante la sesión de interacción de cada bebé participante. ${ }^{*} p<0,05 ; * * p<0,01$ 


\section{Edad y condición de interacción: modelos de regresión lineal}

Se realizaron dos modelos de regresión: uno utilizando como variable dependiente a la frecuencia de miradas dirigidas a la experimentadora por sesión de interacción y el otro utilizando como variable dependiente la duración promedio de cada mirada. En ambos casos las variables independientes utilizadas fueron edad y condición de interacción, tomando el entonamiento afectivo como condición de referencia para el modelo. No fueron incorporadas al modelo la variable sexo, así como las interacciones entre edad y sexo, edad y condición, y condición y sexo, dado que no mostraron ser significativas (Sig. $<0,010$ ). En la tabla 2 se presentan los resultados correspondientes.

Tabla 2

Modelo de análisis de regresión lineal para la frecuencia y la duración promedio de mirada del bebé dirigida a la experimentadora por sesión de interacción. Edad (en meses) y condición de interacción como variables independientes

\begin{tabular}{lccccccc}
\hline & \multicolumn{2}{c}{$\begin{array}{c}\text { Modelo 1. Frecuencia } \\
\text { de mirada (n) }\end{array}$} & $\begin{array}{c}\text { Modelo 2. } \\
\text { Duración } \\
\text { promedio de } \\
\text { mirada (segundos) }\end{array}$ \\
\hline Parámetro & B & SE & Sig. & B & SE & Sig. \\
\hline Intersección & 3,91 & 2,38 & - & 6,28 & 0,54 & - \\
\hline Condición* & & & & & & & \\
\hline Imitación & 5,46 & 1,48 & 0,000 & 0,25 & 0,26 & 0,333 \\
\hline Prototípica & 5,69 & 1,65 & 0,001 & 0,76 & 0,28 & 0,008 \\
\hline $\begin{array}{l}\text { Edad (en } \\
\text { meses) }\end{array}$ & 1,71 & 0,26 & 0,000 & $-0,29$ & 0,05 & 0,000 \\
\hline
\end{tabular}

* Categoría de referencia: entonamiento afectivo.

En ambos modelos, la edad aparece como un factor que afecta significativamente (Sig. 0,000) tanto a la frecuencia como a la duración promedio de mirada, independientemente de las variaciones atribuibles a la condición de interacción. Para el caso de la frecuencia de mirada del bebé dirigida a la experimentadora, se registra un crecimiento de 1,71 miradas por cada mes de aumento en la edad del bebé. En cambio, para la duración promedio, la asociación toma un valor negativo, con un coeficiente de $-0,29$, es decir que la duración promedio de las miradas baja aproximadamente un cuarto de segundo por cada mes de aumento en la edad del bebé.

La condición de interacción con imitación muestra respecto al entonamiento afectivo (categoría de referencia) niveles mayores de frecuencia con un coeficiente de 5,46 (Sig. 0,000), es decir que en la interacción con imitación el bebé suma 5 miradas más a las realizadas en interacción con entonamiento afectivo. En cambio, respecto de la duración promedio, la imitación no muestra diferencias estadísticamente significativas en comparación con la interacción con entonamiento afectivo.

Finalmente, la interacción social prototípica también da cuenta de una asociación positiva entre esta condición de interacción y la frecuencia de mirada (en comparación con el entonamiento afectivo), siendo su coeficiente 5,69 (Sig. 0,001); es decir, en una situación de interacción social prototípica el bebé realiza un poco más de 5 miradas más a las realizadas en la condición de interacción con entonamiento afectivo. Así mismo, la duración de mirada también es mayor en esta condición, con un coeficiente de 0,76 (Sig. 0,008), es decir que la duración promedio de mirada dirigida a la experimentadora en la interacción social prototípica es 0,76 segundos más larga que la duración promedio en la condición de interacción con entonamiento afectivo.

\section{Discusión}

El entonamiento afectivo y la imitación son fenómenos interactivos que implican experiencias intersubjetivas diferentes; sin embargo, según la literatura revisada, hasta el momento no se habían realizado estudios que efectivamente compararan de manera sistemática y evolutiva la reacción social del bebé ante ambas pautas de reciprocidad del 
adulto. El presente trabajo se propuso analizar los efectos que la interacción social con entonamiento afectivo tiene en el involucramiento visual (un indicador de involucramiento social) del bebé en el periodo 6-12 meses, en comparación con la reacción generada por la imitación (la actividad de coincidencia por excelencia) y con la reacción generada en una situación de interacción social prototípica.

Según los resultados descriptivos, encontramos, por un lado, que la condición de interacción con entonamiento afectivo fue la situación de interacción social que generó valores más bajos de involucramiento visual del bebé (en comparación con la situación de interacción con imitación y la interacción social prototípica). Por otro lado, se encontró una tendencia de desarrollo general de la pauta de mirada infantil que se repite en las tres condiciones de interacción: a medida que el bebé crece aumenta la frecuencia y disminuye la duración promedio de la mirada al compañero de interacción.

Para evaluar si la edad y la condición de interacción afectan de manera independiente la pauta de mirada del bebé se llevó adelante un modelo mixto de regresión lineal de los datos obtenidos longitudinalmente sobre los indicadores de mirada de los bebés. Los resultados mostraron que la edad del bebé opera como un factor que afecta a los indicadores del involucramiento visual-social de manera independiente de la condición de interacción social. En línea con los estudios revisados en la introducción, que indican que durante el primer año de vida la mirada del bebé al compañero de interacción se vuelve más móvil e intermitente (D’Odorico \& Levorato, 1990; Kaye \& Fogel, 1980; Messer \& Vietze, 1984; Rochat, 2001 [2004]), los resultados obtenidos en este estudio llevan a pensar que el patrón de desarrollo en la pauta de mirada se mantiene como "telón de fondo" a través de las diferentes interacciones sociales de las cuales el infante esté participando.

Con respecto a los efectos de la condición de interacción, se pudo observar que el entonamiento afectivo provocó un involucramiento visual-social menos intenso que la imitación en términos de frecuencia de mirada dirigida a la experimentadora, pero no hubo diferencias evidentes con respecto a la duración promedio de cada mirada. Es decir, ambas pautas de reciprocidad enmarcadas como actividades de coincidencia efectivamente provocan diferencias en la reacción visual-social del bebé, lo cual brinda confirmación empírica a las propuestas teóricas de los investigadores del área.

Por otro lado, la condición de interacción social prototípica generó un involucramiento visual más intenso que el entonamiento afectivo, tanto en cantidad de veces que los bebés miraron a la experimentadora como en la duración promedio de sus miradas (las cuales fueron casi más de medio segundo más largas que en respuesta al entonamiento afectivo). Esto quiere decir que, con respecto a una interacción que implica pautas de reciprocidad variadas, la situación de entonamiento afectivo provoca menor involucramiento visual del bebé con el compañero de interacción.

Entonces, al comparar la pauta de mirada en las tres condiciones de interacción se hace evidente que, a lo largo del periodo evolutivo estudiado, el entonamiento afectivo fue la pauta de reciprocidad adulta que generó menor involucramiento visual-social. Según los resultados obtenidos, en comparación a una interacción social prototípica y a una interacción con imitación, el entonamiento afectivo genera menos atracción visual hacia el compañero social. Esto podría suponer que el involucramiento intersubjetivo es menor o menos intenso que para las otras condiciones de interacción. Pero esta conclusión sólo es viable si entendiéramos que el contacto social se establece únicamente por medio del contacto visual. Al hacer una reconstrucción más "cualitativa" del comportamiento de la experimentadora en las sesiones de interacción, se vuelve evidente que la pauta de entonamiento afectivo la "obligó" a utilizar pautas de reciprocidad vocales (cuando el bebé se movía) y de contacto físico (cuando el bebé vocalizaba). 
Considerando que tanto el canal auditivo como el canal táctil son vías sensitivas "imposibles de cerrar" (en contraposición con el canal visual), es posible que mientras el bebé se ocupa visualmente de interactuar con otros objetos del mundo - más allá del adulto que lo acompaña - pueda, simultáneamente, estar oyendo o sintiendo en su piel la estimulación que su cuidador le da para acompañar o sintonizar con su comportamiento. Esta reflexión nos lleva a proponer que el entonamiento afectivo podría funcionar como una pauta de reciprocidad adulta que "libera" al bebé del contacto visualsocial, dejando disponible su mirada para explorar o contemplar otros fenómenos de interés en el mundo. Es posible que la coincidencia establecida en la pauta temporal y la intensidad de las acciones permita generar un contacto intersubjetivo que se mantiene más allá del contacto visual. Tal y como sostiene Stern (1985 [1991], 2010), las formas dinámicas de la vitalidad son experiencias afectivas diferentes a las emociones darwinianas básicas, que se expresan en la organización dinámica de las acciones (y no sólo en la expresión facial específica), y son vividas y compartidas a través de distintos canales sensoriales de forma simultánea. De esta manera, no hay razón para que el canal visual funcione como canal de intercambio privilegiado, por lo que el intercambio afectivo puede ocurrir incluso cuando se le "da la espalda" al compañero de interacción. Desde la perspectiva adulta, el entonamiento afectivo podría servir para acompañar las acciones del bebé sin distraerlo; desde la perspectiva del bebé, el entonamiento afectivo podría estar brindándole una experiencia de acompañamiento en sus actividades.

En cambio, la imitación —en comparación con el entonamiento afectivo- mostró ser una actividad de coincidencia que orienta la atención visual del bebé más veces hacia el compañero de interacción. Es decir, en este periodo evolutivo, la coincidencia establecida a nivel global de la acción a través de la imitación parece provocar mayor involucramiento visual de parte del bebé.
Estas diferencias encontradas en la respuesta visual del bebé entre las distintas situaciones de interacción podrían tener ciertas implicancias útiles en el tratamiento clínico de ciertas poblaciones. Por ejemplo, la imitación se ha implementado en el tratamiento de pacientes con autismo y se ha registrado que la imitación materna provoca una mayor orientación de la mirada al rostro de la madre (Dawson \& Galpert, 1990). Esta intervención es considerada saludable, porque la orientación hacia un compañero social es uno de los déficits que caracteriza a los trastornos de espectro autista. Los datos obtenidos con respecto al entonamiento afectivo llevan a preguntarnos qué efectos tendrá esta pauta de reciprocidad en sujetos con estas alteraciones del desarrollo, y abren la puerta a pensar acerca de la efectividad del uso de otros canales sensoriales para la facilitación del establecimiento de un contacto intersubjetivo triádico niño-adultoobjetos del entorno físico.

En síntesis, este trabajo aporta evidencia empírica al estudio del entonamiento afectivo en varios sentidos. Por un lado, se pudo implementar exitosamente una situación experimental que permitió analizar los efectos que el entonamiento afectivo provoca en la reacción social infantil durante la segunda mitad del primer año de vida. De este modo, se obtuvieron datos que permiten cuantificar los efectos diferenciales que las actividades de coincidencia de entonamiento afectivo e imitación provocan en la pauta de mirada del bebé.

Así mismo, se aportaron datos evolutivos (obtenidos longitudinalmente) con respecto a la respuesta visual-social del bebé en la interacción con entonamiento afectivo en el periodo 6-12 meses, contrastándola con la reacción provocada por una situación de interacción social prototípica.

En cuanto a las limitaciones del presente estudio, cabe hacer algunas aclaraciones sobre el modo de registro del material de análisis. Se optó por el registro de las sesiones de interacción con una única cámara fija, la cual genera un material de imagen bidimensional que podría provocar 
algunos desvíos de perspectiva en la observación (Shockley, 2005). Ciertamente podrían obtenerse medidas más precisas de la lectura de la mirada del bebé colocando otras cámaras (dos o cuatro, en lugar de una) que capten al infante desde más ángulos, pero esto haría más dificultoso mantener las condiciones ambientales habituales del niño, lo que podría resultar disruptivo para el desarrollo espontáneo de la interacción en el hogar. Los problemas ocasionados con el modo de registro seleccionado se subsanaron tomando un criterio restringido de categorización observacional en el cual se desestimaron todos los casos en que no podía distinguirse con claridad hacia dónde iba dirigida la mirada del bebé y esto fue contrastado por validez interjueces. En futuros estudios sería interesante, además, medir otros indicadores de involucramiento social del bebé, como la sonrisa, los gestos o el contacto corporal iniciado por el infante; así también sería deseable complementar estos resultados experimentales con nuevos datos sobre la reacción social del bebé obtenidos en contextos naturales de interacción.

\section{Agradecimientos}

Este trabajo fue parcialmente financiado por subsidios otorgados por el Consejo Nacional de Investigaciones Científicas y Técnicas (CONICET) y la Agencia Nacional de Promoción Científica y Tecnológica. Los autores están profundamente agradecidos con los bebés y con las familias que aceptaron participar de este estudio.

\section{Referencias}

Agnetta, B. \& Rochat, P. (2004). Imitative games by 9-, 14-, and 18-month-old infants. Infancy, 6(1), 1-36.

Bateson, M. (1975). Mother-infant exchanges: the epigenesis of conversational interaction. Annals of the New York Academy of Sciences, 263(1), 101-113.
Beebe, B., Rustin, J., Sorter, D., \& Knoblauch, S. (2003). Symposium on intersubjectivity in infant research and its implications for adult treatment. III. An expanded view of forms of intersubjectivity in infancy and its application to psychoanalysis. Psychoanalytic Dialogues, 13(6), 805-841.

Bornstein, M. \& Tamis-LeMonda, C. (2010). Parent-infant interaction. En J. G. Bremner \& T. D. Wachs (Eds.), The Wiley-Blackwell handbook of infant development: Vol. 1. Basic Research (pp. 458-482). Singapur: Blackwell Publishing.

Dawson, G. \& Galpert, L. (1990). Mothers' uses of imitative play for facilitating social responsiveness and toy play in young autistic children. Development and Psychopathology, 2, 151-162.

Di Cesare, G., Di Dio, C., Rochat, M. J., Sinigaglia, C., Bruschweiler-Stern, N., Stern, D. N., \& Rizzolatti, G. (2014). The neural correlates of "vitality form" recognition: an fMRI study. Social cognitive and affective neuroscience, 9(7), 951-960. doi: 10.1093/scan/nst068

D’Odorico, L. \& Levorato, C. (1990). Social and cognitive determinants of mutual gaze between mother and infant. En V. Volterra \& C. J. Erting (Eds.), From gesture to language in hearing and deaf children (pp. 9-17). Nueva York: Springer-Verlag.

Español, S. (2004). Cómo hacer cosas sin palabras. Gestos y ficción en la infancia temprana. Madrid: Antonio Machado.

Español, S. (2012). Semiosis y desarrollo humano. En J. A. Castorina \& M. Carretero (Comps.), Desarrollo cognitivo y educación: Vol.1. Los inicios del conocimiento (pp. 219-240). Buenos Aires: Paidós.

Español, S., Bordoni, M., Carretero, S., Martínez, M., \& Camarasa, R. (2013). Pautas de entonamiento en el juego social entre adulto y bebé. En F. Shifres, M. P. Jacquier, D. Gonnet, M.I. Burcet \& R. Herrera (Eds.), Actas de ECCoM. Vol. 1 $N^{o} 1$, "Nuestro cuerpo en nuestra música. $11^{\circ}$ ECCoM” (pp. 79-86). Buenos Aires: SACCoM. 
Español, S., Martínez, M., Bordoni, M., Camarasa, R., \& Carretero, S. (2014). Forms of Vitality Play in Infancy. Integrative Psychological and Behavioral Science, 48(4), 479-502. doi: 10.1007/ s12124-014-9271-5

Field, T. (1977). Effects of early separation, interactive deficits, and experimental manipulations on infant-mother face-to-face interaction. Child development, 48, 763-771. doi: 10.2307/1128325

Fogel, A. \& Garvey, A. (2007). Alive communication. Infant Behavior \& Development, 30(2), 251257. doi: 10.1016/j.infbeh.2007.02.007

Garvey, A. \& Fogel, A. (2007). Dialogical change processes, emotions, and the early emergence of self. International Journal for Dialogical Science, 2(1), 51-76.

Gonzalez, R., Yu, T., \& Volling, B. (2012). Analysis of experimental and quasi-experimental data: pinpointing explanations. En B. P. Laursen, T. D. Little, \& N. A. Card (Eds.), Handbook of developmental research methods (pp. 247-264). Nueva York: Guilford Press.

Jonsson, C. O. \& Clinton, D. (2006). What do mothers attune to during interactions with their infants? Infant and Child Development, 15(4), 387-402. doi: 10.1002/icd.466

Jonsson, C. O., Clinton, D., Fahrman, M., Mazzaglia, G., Novak, S., \& Sörhus, K. (2001). How do mothers signal shared feeling-states to their infants? An investigation of affect attunement and imitation during the first year of life. Scandinavian Journal of Psychology, 42(4), 377-381. doi: 10.1111/1467-9450.00249

Kaye, K. \& Fogel, A. (1980). The temporal structure of face-to-face communication between mothers and infants. Developmental Psychology, 16(5), 454.

Kipp, M. (mayo, 2008). Spatiotemporal coding in ANVIL. 6th International Conference on Language Resources and Evaluation. Marrakech, Marruecos.

Kugiumutzakis, G. (1998). Neonatal imitation in the intersubjective companion space. En S. Bråten
(Ed.), Intersubjective communication and emotion in early ontogeny (pp. 63-88). Cambridge: Cambridge University Press.

Lewkowicz, D. J. \& Hansen-Tift, A. M. (2012). Infants deploy selective attention to the mouth of a talking face when learning speech. Proceedings of the National Academy of Sciences, 109(5), 1431-1 436. doi: 10.1073/pnas.1114783109

Maratos, O. (abril, 1973). The origin and development of imitation in the first six months of life. British Psychological Association Meeting. Liverpool, Inglaterra.

Maratos, O. (1998). Neonatal, early and later imitation: Same order phenomena? En F. Simion \& G. Butterworth (Eds.), The development of sensory, motor and cognitive capacities in early infancy: from perception to cognition (pp. 145160). East Sussex: Psychology Press.

Markova, G. \& Legerstee, M. (2006). Contingency, imitation, and affect sharing: Foundations of infants' social awareness. Developmental Psychology, 42(1), 132-141.

Meltzoff, A. (1990). Foundations for developing a concept of self: The role of imitation in relating self to other and the value of social mirroring, social modeling, and self practice in infancy. En D. Cicchetti \& M. Beeghly (Eds.), The self in transition: Infancy to childhood (pp. 139-164). Chicago: University of Chicago Press.

Meltzoff, A. (2005 [2010]). Imitación y otras mentes: la hipótesis "como yo" (M. Bordoni, trad.). En D. Pérez, S. Español, L. Skidelsky, \& R. Minervino, (Comps.), Conceptos. Debates contemporáneos en filosofía y psicología (pp. 273-307). Buenos Aires: Catálogos.

Meltzoff, A. \& Moore, M. (1977). Imitation of facial and manual gestures by human neonates. Science, 198, 75-78.

Messer, D. J. \& Vietze, P. M. (1984). Timing and transitions in mother-infant gaze. Infant Behavior and Development, 7(2), 167-181.

Nadel, J. (2006). Does imitation matter to children with autism? En S. Rogers \& J. Williams (Eds.), 
Imitation and the social mind. Autism and typical development (pp. 118-137). Nueva York: The Guilford Press.

Peixoto Junior, C. \& Arán, M. (2011). O lugar da experiência afetiva na gênese dos processos de subjetivação. Psicologia USP, 22(4), 725-746.

Reddy, V. (2008). How infants know minds. Cambridge: Harvard University Press.

Ribas, A. F. P. \& Seidl-de-Moura, M. L. (1999). Manifestações iniciais de trocas interativas mãe-bebê e suas transformações. Estudos de Psicologia (Natal), 4(2), 273-288. doi: 10.1590/S1413294X1999000200005

Richards, J. E., Reynolds, G. D., \& Courage, M. L. (2010). The neural bases of infant attention. Current Directions in Psychological Science, 19(1), 41-46. doi: 10.1177/0963721409360003

Rochat, P. (2001 [2004]). El mundo del bebé (Roc Fillela, trad.). Madrid: Morata.

Rochat, P. \& Striano, T. (2002). Who's in the Mirror? Self-Other Discrimination in Specular Images by Four-and Nine-Month-Old Infants. Child development, 73(1), 35-46.

Sheets-Johnstone, M. (2008). Getting to the heart of emotions and consciousness. En P. Calvo \& A. Gomila (Eds.), Handbook of cognitive science: an embodied approach (pp. 453-463). San Diego: Elsevier.

Shockley, K. (2005). Cross recurrence quantification of interpersonal postural activity. En M. Riley \& G. Van Orden (Eds.), Tutorials in contemporary nonlinear methods for the behavioral sciences (pp. 142-177). Recuperado de http://www.nsf. gov/sbe/bcs/pac/nmbs/nmbs.pdf

Stern, D. (1974). The goal and structure of mother-infant play. Journal of the American Academy of Child Psychiatry, 13(3), 402-421.
Stern, D. (1985 [1991]). El mundo interpersonal del bebé (Jorge Piatigorsky, trad.). Buenos Aires: Paidós.

Stern, D. (1995). The motherhood constellation: a unified view of parent-infant psychotherapy. Nueva York: Karnak books.

Stern, D. (2010). Forms of vitality. Exploring dynamic experience in psychology, the arts, psychotherapy and development. Nueva York: Oxford University Press.

Stern, D., Hofer, L., Haft, W., \& Dore, J. (1985). Affect attunement: the sharing of feeling states between mother and infant by means of inter-modal fluency. En T. Field \& N. Fox (Eds.), Social perception in infants (pp. 249-268). Norwood, NJ: Ablex.

Trevarthen, C. (1998). The concept and foundations of infant intersubjectivity. En S. Bråten (Ed.), Intersubjective Communication and Emotion in Early Ontogeny. (pp. 15-46). Cambridge: Cambridge University Press.

Užgiris, I. (1999). Imitation as activity: its developmental aspects. En J. Nadel \& G. Butterworth (Eds.), Imitation in infancy (pp. 186-206). Cambridge: Cambridge University Press.

Užgiris, I. C., Benson, J. B., Kruper, J. C., \& Vasek, M. E. (1989). Contextual influences on imitative interactions between mothers and infants. En J. J. Lockman \& N. L. Hazen (Eds.), Action in social context: Perspectives on early development (pp. 103-127). Nueva York: Plenum Press.

Villalobos, M. E. (2006). Interacciones tempranas. Envestimiento de sí. Psicología desde el $\mathrm{Ca}$ ribe, $17,60-85$.

Wright, K. (2009). Mirroring and attunement. Self-realization in psychoanalysis and art. Nueva York: Routledge.
Fecha de recepción: enero 31, 2015 Fecha de aceptación: noviembre 10, 2015 
